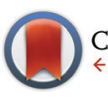

CrossMark \& click for updates

Cite this: Org. Biomol. Chem., 2015, 13, 9789

Received 20th June 2015, Accepted 31st July 2015

DOI: $10.1039 / c 5 o b 01265 a$

www.rsc.org/obc

\section{The interface makes a difference: lanthanide ion coated vesicles hydrolyze phosphodiesters $\uparrow$}

\author{
Michal Poznik, ${ }^{a}$ Uday Maitrab and Burkhard König*a
}

Lanthanide ions are strong Lewis acids. Their complexation to a variety of ligands can further enhance their Lewis acidity allowing the hydrolysis of phosphoesters and even DNA. We show that the interaction of lanthanide ions with vesicles from zwitterionic phosphatidylcholine lipids gives supramolecular structures in which the metal ion is loosely coordinated to the surface. This assembly provides a high density of Lewis-acidic metal centres, which hydrolyze phosphodiesters with enhanced rates.

\section{Introduction}

Phosphodiesters are among the most important functional groups in nature. They form the hydrolytically stable backbone of DNA, which has an estimated half-life of over 30 million years. ${ }^{1}$ Even though high resistance towards degradation and alteration is required for the genetic material, phosphodiesters in DNA are processed by nucleases to read or copy the genetic information. Great efforts have therefore been made to develop artificial systems, which match the specificity and hydrolytic activity of native phosphodiester processing enzymes. ${ }^{2,3}$ Enzymes often contain metal cations in their active centres, which serve as Lewis acids promoting the ester cleavage. The same approach is frequently followed in enzyme mimics $^{4,5}$ and lanthanides are used due to their higher Lewis acidity replacing transition metal ions. ${ }^{6}$ Usually the lanthanide ion is coordinated by a ligand, but the preparation of complexes can be laborious. Simpler self-assembled structures with hydrolytic activity could be advantageous.

We report here the preparation of phospholipid vesicles with lanthanide ions bound to their surface promoting the hydrolysis of phosphodiesters (Fig. 1).

The coordination of metal cations to the surface of bilayers was previously reported. ${ }^{7,8}$ The interaction of europium ions with phospholipids can be followed by changes in emission ${ }^{9}$ and mixtures of cerium cations with surfactants or anionic phospholipids have been reported as promoters of phosphodiester hydrolysis. ${ }^{10-12}$

\footnotetext{
${ }^{a}$ Faculty of Chemistry and Pharmacy, University of Regensburg, 93040 Regensburg, Germany. E-mail: burkhard.koenig@ur.de; Fax: +49 943 1717; Tel: +49 9434576 ${ }^{b}$ Department of Organic Chemistry, Indian Institute of Science, Bangalore, India. E-mail: maitra@orgchem.iisc.ernet.in; Fax: +91-80-2360-0529; Tel: +91-80-2360-1968

$\dagger$ Electronic supplementary information (ESI) available. See DOI: 10.1039/ c5ob01265a
}

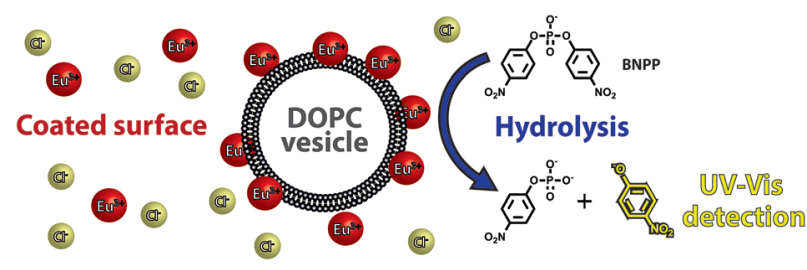

Fig. 1 Phosphodiester hydrolysis with lanthanide ion coated vesicles.

\section{Interaction of metal cations with DOPC vesicles}

We have investigated the interaction of lanthanide cations and $100 \mathrm{~nm}$ vesicles prepared from the zwitterionic lipid 1,2-dioleoyl-sn-glycero-3-phosphocholine (DOPC). In order to achieve high hydrolytic activity of metal complexes it is important to retain their charge in the complex and keep open coordination sites for the interaction with substrates. Negatively charged ligands, such as carboxylates, decrease the overall charge in lanthanide complexes and therefore the hydrolytic activity. In the case of phospholipids the metal ion should interact with several phosphate moieties of the lipid membrane. However, since the vesicles are neutral the overall surface charge will increase by the lanthanide ion binding.

Binding events on the surface of phospholipid vesicles can be monitored by the change of the emission intensity of a coembedded fluorescent dye (Fig. 2). ${ }^{13}$

Amphiphilic carboxyfluorescein (CF) exhibits a strong green fluorescence when embedded in DOPC. The addition of one equiv. of different metal salts with respect to the dye leads to a

\footnotetext{
$¥$ The chloride counter anions will neither tightly coordinate the lanthanide cation in aqueous solution nor bind to the zwitterionic headgroup of the lipid as the formation of an inner-sphere complex between $\mathrm{Eu}^{3+}$ and $\mathrm{Cl}^{-}$is negligible even at high concentrations: F. Tanaka and S. Yamashita, Inorg. Chem., 1984, 23, 2044-2046.
} 


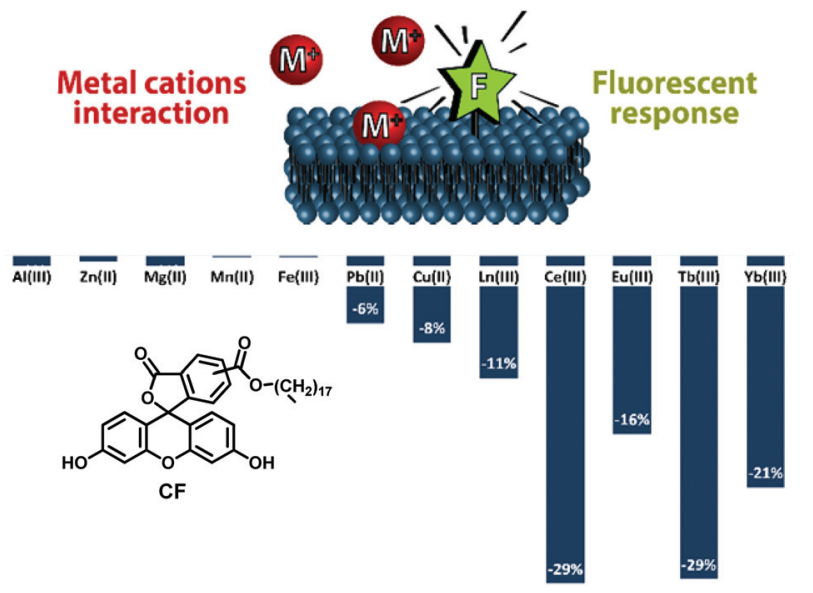

Fig. 2 Effect of different metal ions ( 0.6 equiv. with respect to carboxyfluorescein) on the emission intensity of membrane-embedded carboxyfluorescein $(10 \mathrm{~mol} \%)$ in DOPC vesicles $\left(5 \times 10^{-5} \mathrm{~mol} \mathrm{~L}^{-1}\right)$ in aqueous HEPES buffer at $\mathrm{pH} 7.4$.

decrease in the emission intensity depending on the nature of the metal cation (Fig. 2).

Cations with high charge density induced a larger decrease of the $\mathrm{CF}$ emission intensity. The emission intensity change can be explained by the alteration of the local environment on the surface of the vesicles, most likely due to metal ion binding. The relative change in the emission intensity cannot be used to quantify the amount of metal ion binding to the membrane surface, as emission quenching effects of different metal ions will be very different, but these data provide a qualitative indication of which metal ions interact with the lipid membrane. Under identical conditions, but with non-amphiphilic carboxyfluorescein, the metal ion addition had no effect on the emission intensity (see the ESI†).

Europium chloride has a very characteristic emission spectrum $\left(\lambda_{\text {exc }}=394 \mathrm{~nm}\right)$ whose intensity is strongly influenced by the environment. Intensities of peaks and their ratios provide important information about the coordination around the europium ion. The peak at $615 \mathrm{~nm}$ is known as the hypersensitive transition $\left({ }^{5} \mathrm{D}_{0}-{ }^{-7} \mathrm{~F}_{2}\right)$ and its intensity reflects changes in the ligand binding. ${ }^{14}$ Tracking the intensity changes at $615 \mathrm{~nm}$ compared to other emission maxima allows one to determine differences in ligand coordination. ${ }^{15}$ We observed an increase of the emission peak at $615 \mathrm{~nm}$ when DOPC was added to a buffered solution of europium chloride (see the ESI $\dagger$ ). This effect is likely due to the binding of the europium ion to the phosphate moiety of the lipid.

To confirm the presence of europium ions close to the surface of the membrane, their emission was sensitized using pyrene (Fig. 3). Pyrene has been reported to sensitise effectively the europium phosphorescence in sodium cholate gels. ${ }^{16}$ Pyrene can be located due to its lipophilicity inside the phospholipid bilayer and when irradiated at $365 \mathrm{~nm}$ an increased emission of the europium ions was observed (Fig. 3).
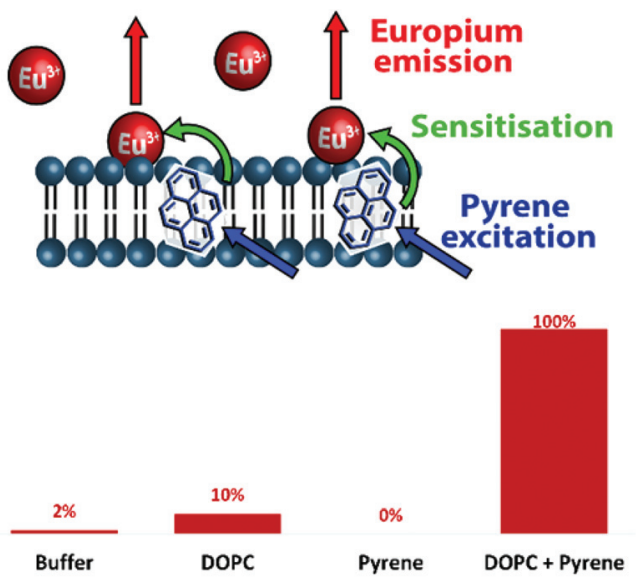

Fig. 3 Sensitization of the emission of membrane-bound europium ions by membrane-incorporated pyrene (top). Relative emission intensity at $615 \mathrm{~nm}$ of $1 \mathrm{mM} \mathrm{EuCl}$ solution in the presence and absence of lipid and pyrene.

The europium ions must therefore be close to the phospholipid membrane. No sensitisation by pyrene was observed in the absence of vesicles.

\section{Hydrolysis of BNPP with different metal ions}

Bis-4-nitrophenyl phosphate (BNPP) was used for investigating the phosphodiester hydrolysis activity of the membranes. The hydrolysis was monitored by the increase in the absorbance at $400 \mathrm{~nm}$ and pseudo first order rate constants were determined by the initial slope method (see the ESI $\dagger$ ). We examined different transition metal ions, which are typically present in hydrolases, and lanthanide ions (Fig. 4). Equimolar amounts of the metal ions as their chloride salts and the lipid in the form of DOPC vesicles were combined, incubated for 2 minutes, then BNPP was added and its hydrolysis was monitored. Identical reactions were performed in the absence of the lipid (Fig. 4). The hydrolytic activity for Fe(III), $\mathrm{Cu}(\mathrm{II}), \mathrm{Zn}$ (II) and $\mathrm{Al}(\mathrm{III})$ is low under these conditions with slight increases in the presence of the lipid. A significantly larger hydrolytic activity

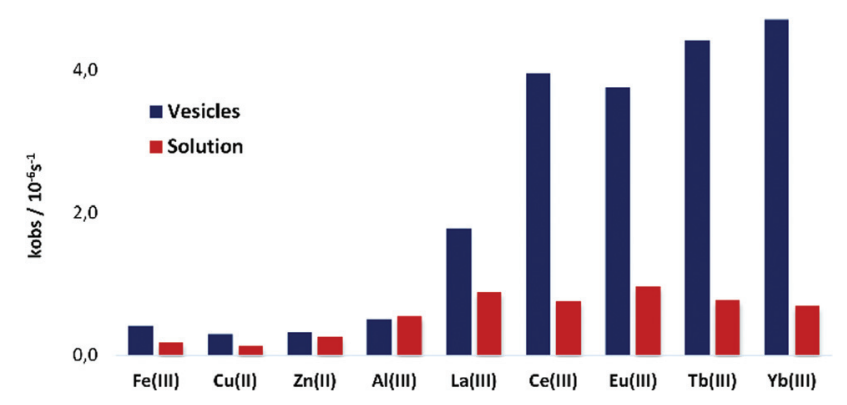

Fig. 4 Pseudo first order rate constants for BNPP hydrolysis in aqueous solution (HEPES, $\mathrm{pH} 7.4$ ) in the presence of different metal ions $(c=$ $10^{-4} \mathrm{~mol} \mathrm{~L}^{-1}$ ) (red) and DOPC ( $c=10^{-4} \mathrm{~mol} \mathrm{~L}^{-1}$ ) (blue) as $100 \mathrm{~nm}$ vesicles. Estimated error of the hydrolysis rate $\pm 5 \%$ from three experiments. 
was observed for lanthanide salts, in particular $\mathrm{Yb}$ (III) and $\mathrm{Tb}$ (III). Here the hydrolytic activity increased up to 7 fold in the presence of the lipid.

\section{Different lipids}

All phospholipids used in this work were zwitterionic phosphatidyl choline lipids varying in length and saturation of the chain, which changes the physical behaviour of the membrane, in particular the transition temperature from the solid (gel) phase to the liquid phase. This transition can greatly affect the properties and behaviour of the embedded amphiphiles, as phase separation and lateral movements change. We examined five different phospholipids (DOPC, DMPC, SMPC, DPPC, DSPC) in the presence of europium ions to investigate the effect of the membrane properties on the hydrolytic activity towards BNPP (for data see the ESI $\dagger$ ). All measurements were conducted at room temperature and all the examined lipids showed similar performance within the error limits of the experiment. Membranes that are in the liquid phase at this temperature gave a slight improvement of about $20 \%$ in the hydrolytic rate and the overall conversion of BNPP after $24 \mathrm{~h}$ (see the $\mathrm{ESI}_{\dagger} \dagger$ ). A rationale for this observation may be the better adaptation of lipids and lanthanide ions to the optimal geometry required for a cooperative BNPP hydrolysis. ${ }^{17}$

\section{Comparison of the hydrolytic properties}

DOPC and europium salts were selected for further investigation. Second order rate constants were determined from the linear regression of pseudo first order rates of the varying europium ion concentration for three ratios of Eu to DOPC: $2: 1$, $1: 1$ and $1: 2$ (see ESI, Fig. S14 $\dagger$ ). The largest rate enhancement of the BNPP hydrolysis was observed for a ratio of Eu : DOPC of $1: 1$. In Table 1 we compare the hydrolytic rates of BNPP under comparable conditions for several lanthanide ion based hydrolysis catalysts. The europium-DOPC vesicles enhance the hydrolytic activity of europium compared to the salt by a factor of 17 and match the rates of simple europium complexes. However, micellar and vesicular systems based on mono and binuclear amphiphilic complexes of cerium(Iv) and zinc show significantly higher rates of BNPP hydrolysis.

Table 1 Comparison of the BNPP hydrolytic activity of Eu : DOPC (1:1) with other metal salts and complexes

\begin{tabular}{llllll}
\hline System & $k_{2}\left(\mathrm{M}^{-1} \mathrm{~s}^{-1}\right)$ & Rel. $k_{2}$ & $T\left({ }^{\circ} \mathrm{C}\right)$ & $\mathrm{pH}$ & Ref. \\
\hline Eu : DOPC $(1: 1)$ & 0.07 & 17 & 25 & 7.4 & $a$ \\
EuCl $_{3}$ & 0.004 & 1 & 25 & 7.4 & $a$ \\
EuDO2A $^{+}$ & 0.017 & & 25 & 9.35 & 18 \\
Eu (THED) & 0.11 & & 37 & 7.5 & 19 \\
Eu : peptide & $0.1-0.3$ & & 37 & 7.7 & 20 \\
Ce(Iv)-micelles & 0.6 & & 37 & 7 & 21 \\
Bis-Zn-cyclen micelles & 7.9 & & 25 & 7.4 & 22 \\
Bis-Zn-cyclen : DOPC & 9.4 & & & & \\
& & & & &
\end{tabular}

\section{Conclusions}

Lanthanide cations bind to the lipid-water interface of DOPC vesicles. The interaction of metal ions with the membrane was confirmed by induced changes of the spectroscopic properties of the membrane embedded carboxyfluorescein. The sensitisation of the europium emission by the membrane embedded pyrene verifies its close proximity to the interface. The higher local concentration of the lanthanide ions at the lipid interface is a likely rationale for the increased hydrolytic activity towards the activated phosphodiester BNPP by a factor of 17 compared to aqueous europium salts. Such hydrolytically active membranes may be used to coat objects or surfaces providing them with chemical reactivity. Their potential applications are in the processing of biomolecules.

\section{Acknowledgements}

We thank Raju Laishram for experimental support. UM thanks the Department of Science \& Technology, New Delhi for the award of a J. C. Bose Fellowship.

\section{Notes and references}

1 G. K. Schroeder, C. Lad, P. Wyman, N. H. Williams and R. Wolfenden, Proc. Natl. Acad. Sci. U. S. A., 2006, 103, 4052-4055.

2 M. A. Zenkova, Artificial Nucleases, Springer-Verlag, Berlin, Heidelberg, 2004.

3 J. A. Cowan, Curr. Opin. Chem. Biol., 2001, 5, 634-642.

4 F. Mancin, P. Scrimin, P. Tecilla and U. Tonellato, Coord. Chem. Rev., 2009, 253, 2150-2165.

5 F. Mancin, P. Scrimin and P. Tecilla, Chem. Commun., 2012, 48, 5545-5559.

6 S. J. Franklin, Curr. Opin. Chem. Biol., 2001, 5, 201-208.

7 M. N. Jones, K. Hammond, M. D. Reboiras, C. Acerete, S. M. Jackson, M. Nogueira and A. R. Nicholas, Colloids Surf., A, 1986, 18, 75-91.

8 C. Altenbach and J. Seelig, Biochemistry, 1984, 23, 39133920.

9 N.-E. L. Saris, Chem. Phys. Lipids, 1983, 34, 1-5.

10 K. Bracken, R. A. Moss and K. G. Ragunathan, J. Am. Chem. Soc., 1997, 119, 9323-9324.

11 N. Kimizuka, E. Watanabe and T. Kunitake, Chem. Lett., 1999, 28, 29-30.

12 J. Sumaoka, T. Igawa, K. Furuki and M. Komiyama, Chem. Lett., 2000, 29, 56-57.

13 B. Gruber, S. Stadlbauer, K. Woinaroschy and B. Konig, Org. Biomol. Chem., 2010, 8, 3704-3714.

14 S. V. Eliseeva and J.-C. G. Bunzli, Chem. Soc. Rev., 2010, 39, 189-227.

15 P. K. Gallagher, J. Chem. Phys., 1964, 41, 3061-3069.

16 S. Bhowmik, S. Banerjee and U. Maitra, Chem. Commun., 2010, 46, 8642-8644. 
17 M. Poznik and B. Koenig, Org. Biomol. Chem., 2014, 12, 20 J. T. Welch, M. Sirish, K. M. Lindstrom and S. J. Franklin, 3175-3180. Inorg. Chem., 2001, 40, 1982-1984.

18 C. A. Chang, B. H. Wu and B. Y. Kuan, Inorg. Chem., 2005, 21 R. Bonomi, P. Scrimin and F. Mancin, Org. Biomol. Chem., 44, 6646-6654. $2010,8,2622-2626$.

19 J. R. Morrow, K. Aures and D. Epstein, J. Chem. Soc., Chem. 22 B. Gruber, E. Kataev, J. Aschenbrenner, S. Stadlbauer and Commun., 1995, 2431-2432. B. König, J. Am. Chem. Soc., 2011, 133, 20704-20707. 